\title{
Local excision followed by early radical surgery in rectal cancer: long-term outcome
}

\author{
Theodor Junginger ${ }^{1}$, Ursula Goenner ${ }^{1}$, Mirjam Hitzler ${ }^{2}$, Tong T. Trinh ${ }^{3}$, Achim Heintz ${ }^{2}$ and Daniel Wollschläger ${ }^{4^{*}}$ (D)
}

\begin{abstract}
Background: In rectal cancers, radical surgery should follow local excisions, in cases of unexpected, unfavorable tumor characteristics. The oncological results of this completion surgery are inconsistent.

This retrospective cohort study assessed the clinical and long-term oncological outcomes of patients that underwent completion surgery to clarify whether a local excision compromised the results of radical surgery.
\end{abstract}

Methods: Forty-six patients were included, and the reasons for completion surgery, intraoperative complications, residual tumors, local recurrences (LRs), distant metastases, and cancer-specific survival (CSS) were assessed. The results were compared to 583 patients that underwent primary surgery without adjuvant therapy, treated with a curative intention during the same time period.

Results: The median follow-up was 14.6 years. The reasons for undergoing completion surgery were positive resection margins (24\%), high-risk cancer (30\%), or both (46\%). Intraoperative perforations occurred in 10/46 (22\%) cases. Residual tumor in the rectal wall or lymph node involvement occurred in 12/46 (26\%) cases. The risk of intraoperative perforation and residual tumor increased with the pT category. Intraoperative perforations did not increase postoperative complications, but they increased the risk of LRs in cases of intramural residual tumors $(p=0.003)$. LRs occurred in $2.6 \%$ of pT1/2 and $29 \%$ of pT3 tumors. Both the 5- and 10-year CSS rates were $88.8 \%$ (95\% Cl 80.0-98.6). Moreover, the LRs of patients with pT1/2 cancers were lower in patients with completion surgery than in patients with primary surgery.

Conclusions: Rectal wall perforations at the local excision site and residual cancer were the main risks for poor oncological outcomes associated with completion surgery. Local excisions followed by early radical surgery did not appear to compromise outcomes compared to patients with primary surgery for pT1/2 rectal cancer. Improvements in clinical staging should allow more appropriate selection of patients that are eligible for a local excision of rectal cancer.

Keywords: Rectal cancer, Local excision, Completion surgery, Intraoperative perforation, Oncological outcome

\section{Introduction}

A local excision is an alternative to radical surgery in patients with low-risk rectal carcinoma [1], and it is increasingly used when patients show good responses to chemoradiotherapy (CRT) [2]. Local excision provides several advantages over radical surgery; for example, it provides lower morbidity and mortality [3]; it carries a lower risk of functional disorders, because it preserves the rectum

\footnotetext{
* Correspondence: wollschlaeger@uni-mainz.de

${ }^{4}$ Institute of Medical Biostatistics, Epidemiology and Informatics, University Medical Centre of the Johannes Gutenberg-University, Langenbeckstr. 1, D 55131 Mainz, Germany

Full list of author information is available at the end of the article
}

and anal sphincters [4]; and it avoids an abdomino-perineal resection (APR), which is associated with a permanent colostomy in patients with low rectal cancer.

Increasingly, flexible endoscopy with a submucosal dissection has permitted a surgical-like en bloc resection of superficial rectal lesions [5]. An assessment of the resected specimen can reveal tumor characteristics that determine the risk of lymph node metastases or positive resection margins, which carry a high risk of local recurrence (LR). Early radical surgery can lower the risk of LR [6], and it offers a better prognosis than salvage surgery, in cases of clinically manifest LR [7]. However, evidence

(C) The Author(s). 2019 Open Access This article is distributed under the terms of the Creative Commons Attribution 4.0 International License (http://creativecommons.org/licenses/by/4.0/), which permits unrestricted use, distribution, and 
on radical surgery strategies and its results has been inconclusive, mainly because we lack large data sets and long follow-up periods. Moreover, there is no consensus about the optimal timing of radical surgery.

A primary problem with this approach is the risk of intraoperative perforation at the local excision site, which might impact the surgical treatment and the outcome. It remains unclear whether an intraoperative perforation raises the risks of postoperative morbidity $[8,9]$ or the risk of requiring an APR $[10,11]$. In addition, it is not clear whether intraoperative perforations compromise oncological outcomes. Furthermore, it is not known whether applying radiotherapy (RT) [11] or CRT [12] before radical surgery might provide any benefit. Randomized studies are lacking.

Therefore, in this study, we performed a retrospective analysis of the results of local excision followed by radical surgery and compared them to the results of primary conventional surgery. This study aimed to (a) investigate the influence of residual cancer and intraoperative perforations at the site of local excision on postoperative clinical and long-term oncological outcomes and (b) clarify whether the oncological results of radical surgery after local excision were inferior to the results of primary conventional surgery for patients treated within the same time period.

\section{Patients and methods}

All patients with rectal cancer were registered in a prospectively maintained database at the Department of General and Abdominal Surgery of the University Medical Center, Mainz (Germany). We identified patients that had received radical completion surgery after a local excision for rectal cancer from 1985 to 2007. Before the local excision was performed, the preoperative diagnosis workup included a digital rectal examination, rigid rectoscopy, endorectal ultrasonography, and abdominal computed tomography. Magnet resonance imaging (MRI) was not routinely performed at the beginning of the study period. The preferred method of local excision was transanal endoscopic full-thickness microsurgery (TEM), with closure of the defect in the rectal wall [13]. Very low tumors were removed with a transanal excision (TAE), performed with a Parks retractor. The indications for a local excision of rectal cancer changed during the study period. When TEM was first introduced in our institution (i.e., the beginning of the TEM era), uT2 tumors were included, in cases of favorable tumor characteristics. Later on, these patients were excluded, according to existing guidelines.

The reasons for early radical surgery were retrospectively classified as follows: (1) a defective local excision (positive margins), which included incomplete resections (R1), unclear margins, or unsafe resection margins (i.e., $\leq$
$1 \mathrm{~mm}$ between the tumor and the deep or lateral resection margin); (2) unfavorable tumor characteristics that indicated high-risk carcinoma, including pT2/3 tumors [14], lymphovascular invasion (L1) [15], perineural invasion, or poor differentiation (G3/4) [16]; or (3) both a defective excision and high-risk carcinoma characteristics.

We collected data on demographic characteristics: the size and location of the tumor in the rectum (locations were based on the distance from the anal verge, as follows: lower third, $0-6.0 \mathrm{~cm}$; middle third, $6.1-12.0 \mathrm{~cm}$; and upper third, $12.1-16 \mathrm{~cm}$ ); the type of local excision (TEM/TAE, full-thickness/partial resection); the time between the local excision and radical surgery; the reason for radical surgery; intraoperative complications, particularly perforation of the rectal wall; the type of radical surgery (sphincter-saving procedure, low anterior or anterior resection, or APR); postoperative morbidity; and the final pathology assessment [17].

Patients were followed up with regular visits to the oncology department of the hospital, according to a standardized program, until the fifth postoperative year following hospital discharge. Follow-up data were updated for all patients, including those with LR or distant metastasis (DM), in 2012, and 2017. Follow-ups were conducted by contacting the patients, their families, treating physicians, and hospitals. These data included vital status, the presence/absence of disease, the results of follow-up visits, the dates and treatments of tumor recurrences, and the date and cause of death, when applicable.

Local control was determined between the time of radical surgery and a confirmed LR. LR was defined as clinical, radiological, or histological evidence of a recurrent tumor in the local excision site or pelvis, irrespective of DM. DMs were defined as radiological evidence of tumor spread, with or without a LR.

Cancer-specific survival (CSS) was defined as the time from the local excision to death due to rectal cancer. Patients that died from other causes were censored at the time of death. Only tumor recurrence at the time of death and death after surgery due to recurrence were considered events for CSS determinations.

Of 185 patients treated with TEM due to rectal cancer, $42(22.7 \%)$ received completion surgery. Of these 42 patients, 4 were excluded because the radical resection was performed immediately after an intraoperative pathological assessment of the specimen resected with TEM (these 4 patients did not experience LR). Of 28 patients treated with TAE, 8 (28.6\%) underwent early radical surgery. Consequently, the present study included 46 patients that received local excisions, followed by early radical surgery, then follow-up examinations, until December 31, 2017. Short-term oncological results of fewer patients were published previously [18]. 
To compare the oncological outcomes of local excision followed by radical surgery to those of conventional treatment, we identified 583 consecutive patients with pT1-3 rectal cancer that received primary surgery with a curative intention (R0 resection) from 1985 to 2007. These patients did not receive adjuvant therapy. Patients were followed until December 31, 2012.

The total mesorectal excision (TME) technique was introduced in 1996. Thus, both groups included patients with and without TMEs. For comparisons, we reported the results of LRs that occurred after surgeries with and without a TME, in both groups of patients.

\section{Statistical analysis}

Patient characteristics are described as the percentage, median, or mean values. Differences among subgroups of patients with and without LR were assessed with the chi-square test or Fisher's exact test, as appropriate, for categorical outcomes, and with the Kruskal-Wallis test for continuous outcomes. $p$ values were unadjusted for multiple testing. $p$ values or confidence intervals (CIs) are presented in the text and tables. The Kaplan-Meier method was used to estimate the probability of CSS over time. Survival times were compared between groups with the log-rank test (univariate analysis). All statistical analyses were performed with the $\mathrm{R}$ environment for statistical computing, version 3.5.1 [19].

\section{Results}

Table 1 shows the characteristics of 46 patients that underwent a local excision followed by radical surgery. Table 2 shows the reasons for early completion surgery. TEM was performed in 38/46 (83\%) patients. Rectal cancer was located in the middle third of the rectum in most of these patients, but all tumors removed with TAE were located in the lower third of the rectum. In some patients with rectal cancer in the upper third, the rectal wall was partially resected with TEM to avoid opening the peritoneal cavity. In patients with very low rectal cancers, rectal wall was incompletely resected in the region of anal sphincters to avoid injury. The median time interval between the local excision and radical surgery was 21 (range 7-86) days. In 93\% (43/46) of patients, radical surgery was performed within 42 days.

\section{Radical surgery}

All 46 patients received open, non-laparoscopic resections. Of these, 35 (76\%) were sphincter-saving procedures, including 2 anterior resections and 33 low anterior resections; of the latter, 8 received hand-sewn coloanal anastomoses. Twenty-two patients received conventional radical surgery without a formal TME, and 24 patients received early TME surgery. In 11 patients (24\%), APRs were performed with a permanent
Table 1 Characteristics of the 46 patients with rectal cancer treated with local excision followed by radical surgery

\begin{tabular}{|c|c|c|c|}
\hline & & \multicolumn{2}{|c|}{ Pathological characteristics } \\
\hline Median & 64.3 & pT1 & $16(35 \%)$ \\
\hline \multirow[t]{2}{*}{ Range } & $33.6-78.0$ & pT2 & $23(50 \%)$ \\
\hline & & pT3 & $7(15 \%)$ \\
\hline \multirow[t]{3}{*}{ Male/female } & $27 / 19$ & & \\
\hline & & pNO & 37 (80\%) \\
\hline & & pN1 & $7(15 \%)$ \\
\hline Tumor size $(\mathrm{cm})$ & & $\mathrm{pN} 2$ & $2(4 \%)$ \\
\hline Median & 2.8 & & \\
\hline \multirow[t]{3}{*}{ Range } & $0.7-6$ & $\mathrm{pT} 1 \mathrm{~N}+$ & $1(6 \%)$ \\
\hline & & pT2N+ & $4(17 \%)$ \\
\hline & & pT3N+ & $4(57 \%)$ \\
\hline \multicolumn{4}{|l|}{ Tumor site } \\
\hline Lower third & $8(18 \%)$ & Grading & \\
\hline Middle third & $22(48 \%)$ & $\mathrm{G} 1 / 2$ & $35(76 \%)$ \\
\hline Upper third & $16(34 \%)$ & $\mathrm{G} 3 / 4$ & $11(24 \%)$ \\
\hline \multicolumn{2}{|l|}{ Type of resection } & \multicolumn{2}{|c|}{ Lymphovascular invasion } \\
\hline TEM & $38(83 \%)$ & LO & $4(9 \%)$ \\
\hline Full thickness & 28 & L1 & $7(15 \%)$ \\
\hline Partial & 10 & $L X$ & $35(76 \%)$ \\
\hline TAE & $8(17 \%)$ & & \\
\hline Full thickness & 1 & & \\
\hline Partial & 6 & & \\
\hline Unclear & 1 & & \\
\hline
\end{tabular}

colostomy. The percentage of APRs performed depended on tumor location: $59 \%$ (10/17) were performed for tumors in the lower third, $4 \%$ were performed $(1 / 23)$ for tumors in the middle third, and none $(0 / 6)$ were performed for tumors in the upper third. APRs were performed significantly more frequently after a TAE than after a TEM $(7 / 8,88 \%$ vs. $4 / 38$, $11 \%, p<0.001)$. Intraoperative perforations of the rectal wall at the local excision sites were observed in $10 / 46(22 \%)$ patients, and the rate was higher among patients with $\mathrm{pT}$ categories ( $p=0.03$; Table 3$)$. The rate of rectal wall perforation was higher with a full-thickness resection $(n=8 / 29,28 \%)$ than with a partial resection $(n=1 / 16,6 \% ; p=0.1)$. Intraoperative perforations did not significantly increase the frequency of APRs: APRs were performed in 3/10 (30\%) patients with and $8 / 36(22 \%)$ patients without intraoperative perforations $(p=0.7)$.

The postoperative course was uneventful in 36 of 46 patients $(78 \%)$. Ten patients $(22 \%)$ experienced postoperative complications. There was no postoperative death. 
Table 2 Causes for early radical surgery following local excision of rectal cancer and residual cancer in resected specimen of 12 patients

\begin{tabular}{|c|c|c|c|}
\hline \multicolumn{2}{|l|}{ Cause of early radical surgery } & \multirow{2}{*}{$\begin{array}{l}\text { Residual tumor in rectal wall } \\
6 * / 46(13 \%)\end{array}$} & \multirow{2}{*}{$\begin{array}{l}\text { Lymph node metastases } \\
9 * / 46(20 \%)\end{array}$} \\
\hline$n$ & 46 & & \\
\hline \multicolumn{4}{|l|}{ Positive resection } \\
\hline Margin $^{* *}$ & $11(24 \%)$ & 0 & 0 \\
\hline High-risk carcinoma & $14(30 \%)$ & 0 & $3(21 \%)$ \\
\hline \multicolumn{4}{|l|}{ Positive resection } \\
\hline \multirow[t]{2}{*}{ Margin and high-risk carcinoma** } & $21(46 \%)$ & $6^{*}(29 \%)$ & $6^{*}(29 \%)$ \\
\hline & & $p=0.02$ & $p=0.055$ \\
\hline
\end{tabular}

*3 patients had residual tumor in rectal wall and involved nodes

**Incomplete resection (R1) or indeterminate or unsafe resection margin (minimal distance $\leq 1 \mathrm{~mm}$ )

Four of the 35 (11\%) patients that received sphincterpreserving procedures experienced anastomotic leakage. An intraoperative tear of the rectal wall at the site of local excision had no influence on the rate of postoperative complications ( $20 \%$ with and $22 \%$ without intraoperative tears).

\section{Residual carcinoma}

All resected specimens after radical surgery were classified as R0 resections. Seven patients had pT3 tumors (Table 1 ). Of these, 4 were clinically classified as uT2 and 3 were classified as uT0-1 tumors, based on endorectal sonography before the local excision. In 2 patients (uT0-1), pathological examinations of the local excision revealed a pT1 carcinoma, but perirectal infiltration (pT3) was detected in the resected specimen after radical surgery. Intramural tumors and/or involved lymph nodes were observed in 12/46 (26\%) patients.

Among the different reasons for radical surgery, patients with positive resection margins combined with high-risk carcinoma had the highest frequency of residual cancer tissue $(9 / 21,43 \%, p=0.008$, Table 2). Residual cancer in the rectal wall was detected in $6 / 46$ (13\%) patients, and the frequency increased with the degree of tumor infiltration ( $p=0.01$, Table 3). Residual cancer occurred more often after TAE than after TEM $(3 / 8,38 \%$ vs. $3 / 38,8 \%, p=0.06)$.

Table 3 Intraoperative perforation, residual tumor and local recurrence of 46 patients with early radical surgery after local excision of rectal carcinoma

\begin{tabular}{llllll}
\hline & al & pT1 & pT2 & pT3 & $p$ \\
& 46 & 16 & 23 & 7 & \\
\hline Tumor size (cm) (median) & 2.8 & 2.4 & 3.0 & 3.0 & \\
Intraoperative perforation & $10(22 \%)$ & 0 & $7(30 \%)$ & $3(43 \%)$ & 0.03 \\
Residual tumor rectal wall & $6(13 \%)$ & 0 & $3(13 \%)$ & $3(43 \%)$ & 0.01 \\
Lymph node metastases & $9(20 \%)$ & $1(6 \%)$ & $4(17 \%)$ & $4(57 \%)$ & 0.028 \\
Local recurrence & $3(7 \%)$ & $1(6 \%)$ & 0 & $2(29 \%)$ & 0.03 \\
\hline
\end{tabular}

In 9 of 46 patients (20\%), lymph nodes were involved (N1: $n=7, \mathrm{~N} 2: n=2)$. Similar to the incidence of residual tumors in the rectal wall, the incidence of lymph node metastases increased with the pT category $(p=0.028$, Table 3$)$. Of the 9 patients with involved nodes, 3 received postoperative adjuvant $\mathrm{CRT}$ and 2 received adjuvant chemotherapy.

\section{Local recurrence}

The median follow-up times were 14.7 years for patients that received early conventional surgery and 11.6 years for patients that received early TME surgery. Among patients that survived throughout the study, the median follow-up time was 16.5 years (range 8.6-20.6). LR was observed in $3 / 46$ patients $(6.5 \%)$, at $0.7,2.9$, and 3.3 years after radical surgery. In all 3 patients, the causes for early radical surgery were incomplete resection (R1) combined with high-risk carcinoma. Two patients with LR had received conventional surgery and 1 had received TME surgery (Table 4). A univariate analysis identified four significant risk factors for LR: the $\mathrm{pT}$ category $(p=0.03)$; the $\mathrm{pN}$ category $(p=0.04)$; a residual tumor in the rectal wall and involved lymph nodes $(p=0.02)$; and a perforation in the rectal wall, in cases of residual wall tumor $(p=0.003)$. After an intraoperative perforation, LR was observed in both patients with residual tumors in the rectal wall, but not in 8 patients without residual tumors. A multivariate analysis could not be performed, due to the small number of events.

\section{Distant metastasis}

In 6 of $46(13 \%)$ patients, DMs were observed with $(n=1)$ or without $(n=5)$ LR. The incidence of DMs significantly increased with the $\mathrm{pN}$ category $(p=0.02)$, but it was not significantly related to the $\mathrm{pT}$ category.

\section{Survival}

At the end of follow-up, 19 of 46 patients (41\%) had died. Six deaths were due to rectal cancer, and 13 were due to unrelated diseases. Both the 5- and 10-year CSS rates were $88.0 \%$ (95\% CI 80.0-98.6; Fig. 1). Both the 5- 
Table 4 Local recurrences in patients with primary surgery and patients with local excision followed by radical surgery separated for conventional and TME-surgery (pT1-pT3 cancer of the rectum). The differences within conventional surgery $(p=0.2)$ and TME surgery $(p=0.7)$ were not significant

\begin{tabular}{|c|c|c|c|c|c|c|c|c|c|c|c|c|}
\hline & \multicolumn{6}{|c|}{ Conventional surgery } & \multicolumn{6}{|c|}{ TME-surgery } \\
\hline & \multicolumn{3}{|c|}{ Primary surgery } & \multicolumn{3}{|c|}{ LE followed by RS } & \multicolumn{3}{|c|}{ Primary TME-surgery } & \multicolumn{3}{|c|}{ LE followed by TME } \\
\hline & Number & $\operatorname{LR}(n)$ & Percent & Number & $\operatorname{LR}(n)$ & Percent & Number & $\operatorname{LR}(n)$ & Percent & Number & $\operatorname{LR}(n)$ & Percent \\
\hline pT1No & 39 & 1 & 2.3 & 11 & 1 & 9.1 & 33 & 0 & 0 & 4 & 0 & 0 \\
\hline pT1N+ & 4 & 0 & & 0 & 0 & & 2 & 0 & & 1 & 0 & \\
\hline pT2NO & 96 & 11 & 19.5 & 7 & 0 & 0 & 97 & 2 & 1.9 & 12 & 0 & 0 \\
\hline pT2N+ & 30 & 6 & & 2 & 0 & & 9 & 0 & & 2 & 0 & \\
\hline $\mathrm{pT} 1 / 2 \mathrm{NO}$ & 135 & 12 & 10.7 & 18 & 1 & 5.0 & 130 & 2 & 1.4 & 16 & 0 & 0 \\
\hline $\mathrm{pT} 1 / 2 \mathrm{~N}+$ & 34 & 6 & & 2 & 0 & & 11 & 0 & & 3 & 0 & \\
\hline pT3NO & 103 & 22 & 22.6 & - & - & 50.0 & 78 & 10 & 14.2 & 3 & 0 & 20 \\
\hline pT3N+ & 56 & 14 & & 2 & 1 & & 35 & 6 & & 2 & 1 & \\
\hline
\end{tabular}

LE Local excision, TME Total mesorectal excision, RS Radical surgery, LR Local recurrence

and 10-year CSS rates were significantly reduced in patients with involved lymph nodes $(p<0.001)$, proven residual tumors $(p=0.008)$, and $\mathrm{pT}$ category tumors $(p=0.03$; Fig. $2 \mathrm{a}-\mathrm{c})$.

\section{Comparison with primary surgery}

Out of 583 patients with pT1-3, N0, and N+ rectal cancers that received primary R0 resections, 330 (56.6\%) received conventional resections and 253 (43.4\%) received TMEs. Inadvertent perforations were observed in 24/583 (4.1\%) patients. Sphincter saving procedures were performed in $403 / 583(69.1 \%)$ patients. The median follow-up was 72 months.

LRs were higher after conventional surgery than after TME surgery. In patients with pT1/2 cancers, LRs occurred less frequently after a local excision followed by

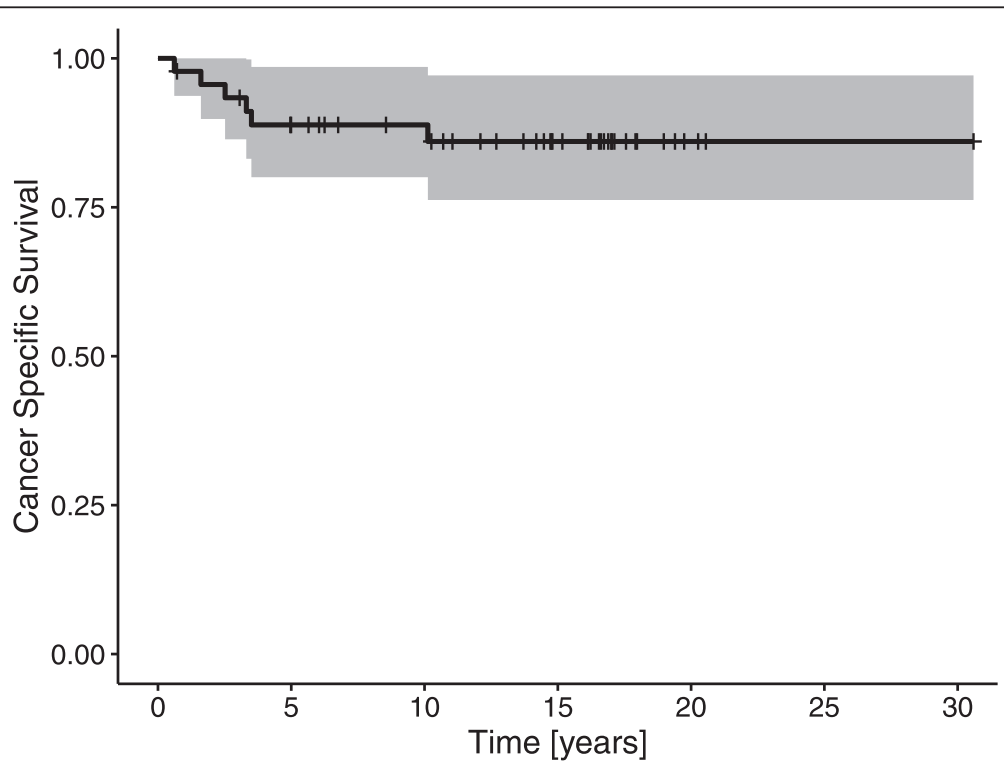

Number at risk

\begin{tabular}{|c|c|c|c|c|c|c|c|}
\hline 氶 All & 46 & 37 & 32 & 20 & 3 & 1 & 1 \\
\hline & 0 & 5 & 10 & $\begin{array}{r}15 \\
\text { e [y }\end{array}$ & 20 & 25 & 30 \\
\hline
\end{tabular}

Fig. 1 Kaplan-Meier survival analyses show cancer-specific survival for all 46 patients with rectal cancer that received a local excision followed by radical surgery 

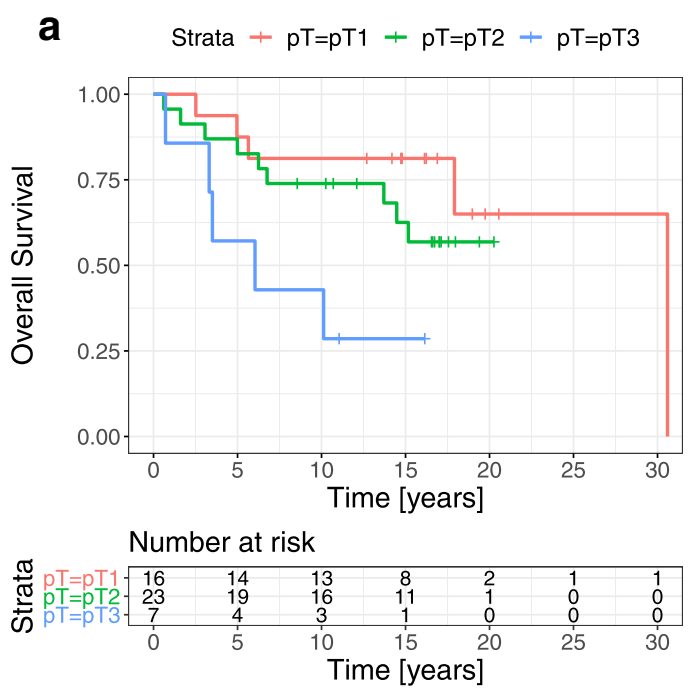

b $\quad$ Strata $+\mathrm{pN}=\mathrm{pNO}+\mathrm{pN}=\mathrm{pN} 1+\mathrm{pN}=\mathrm{pN} 2$
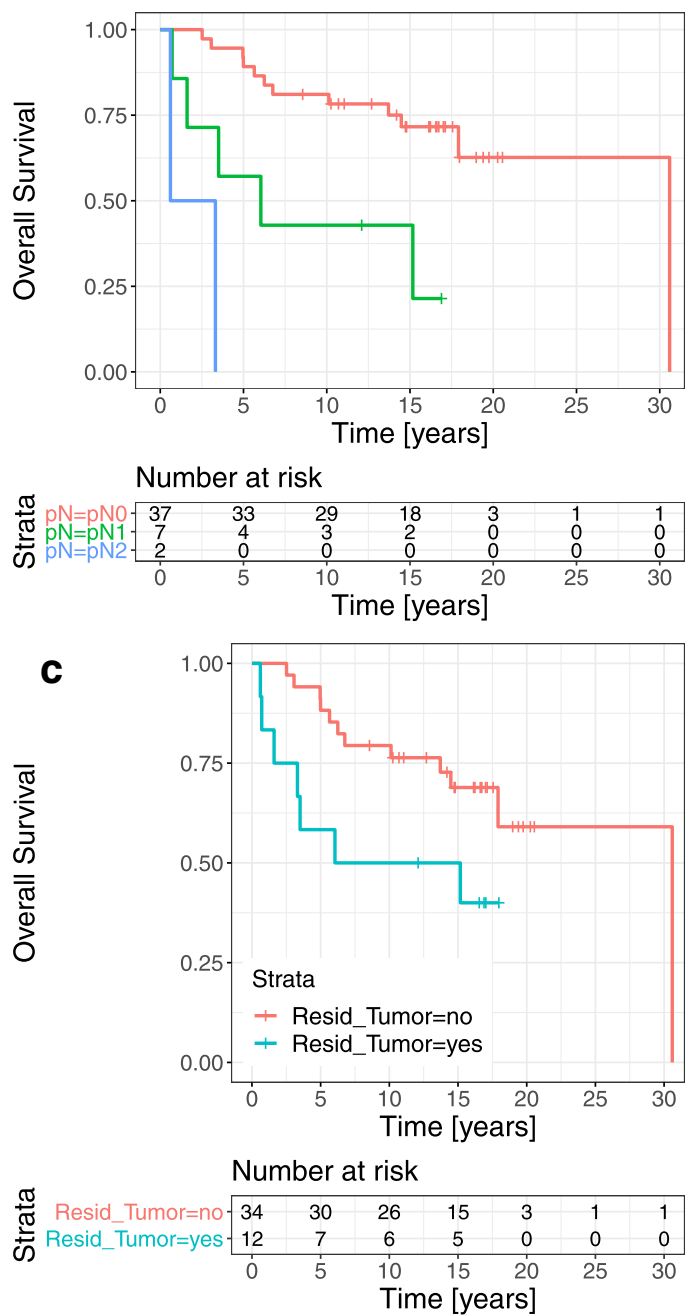

Fig. 2 Cancer-specific survival, grouped according to disease category. Survival curves are shown for patients, according to a pT categories $(p=0.03)$, $\mathbf{b}$ pN categories $(p<0.001)$, and $\mathbf{c}$ the detection of residual intramural tumors and/or lymph node metastases $(p=0.008)$

surgery than after primary surgery. No LR was observed after LE followed by TME. Conversely, in patients with pT3 cancer, LRs occurred more frequently after local excision followed by surgery than after primary surgery. However, the differences were not significant (Table 4).

\section{Discussion}

Patients with positive margins after local excisions, combined with high-risk carcinoma, had the highest rates of residual cancer at completion surgery. The rates of residual intramural tumor tissue and involved lymph nodes increased with the pT category. An intraoperative opening at the local excision site increased the risk of $\mathrm{LR}$, in cases of residual intramural tumor. The oncological results were in the range of the results of patients that received primary surgery.

There is a general consensus that local excisions, preferably a TEM [20] or transanal minimally invasive microsurgery (TAMIS) [21], are an acceptable alternative to conventional radical surgery for patients with low-risk pT1 rectal carcinoma [1]. Furthermore, it is accepted that radical surgery should follow local excisions, in cases of unexpected unfavorable tumor characteristics [22]. However, radical surgery has some challenging aspects. On one hand, the residual cancer should be eradicated as soon as possible, but, on the other hand, an early second surgical procedure might increase the risk of complications. The rectal wall is weakened after a local excision, and tearing during traction might allow cancer cells to spill out. Moreover, fibrotic scars and adhesions between the mesorectum and the pelvic wall might hinder dissections in the correct plane, and thus, the quality of the resected specimen could be limited [23]. Depending on how these trade-offs were managed, the time between a local excision and radical surgery has varied broadly, from 7 days [24] to 15 weeks [25], in previously reported series. In the present study, early radical surgery was performed within 21 days (median) after a local excision.

A special issue associated with completion surgery after a local excision is the high risk of intraoperative perforation at the local excision site, which may affect the clinical and oncological outcomes. Piessen et al. [8] hypothesized that the practice of leaving the defect unsutured after a TAE contributed to both the high rate of rectal wall tearing (36\%) during completion surgery and the high rate of post-surgical complications. They hypothesized that closing the defect would minimize 
these complications. In the present study, the defect from a TEM was routinely closed after a full-thickness resection. Nevertheless, the rate of intraoperative rectal wall opening was only marginally lower (28\%) than that observed by Piessen et al. [8], and it was consistent with the rates observed in other studies on completion surgery [23, 24]. Van Gijn et al. [11] regularly observed perforations during TME following a TEM. A lower perforation rate $(6 \%)$ might be expected after a partial resection of the muscularis propria, but this approach is not acceptable when a local excision is planned as the only treatment for early rectal carcinoma.

In the present study, intraoperative perforations did not influence the risk of postoperative complications, but they influenced the risk of LR, when residual cancer was present in the rectal wall. It is well known that an intraoperative tumor perforation is a risk factor for LR in patients that undergo primary surgery for rectal cancer [26]. That finding was confirmed in the present study: residual intramural tumors and intraoperative perforations were the most significant risk factors for $\mathrm{LR}$, apart from the $\mathrm{pT}$ and $\mathrm{pN}$ categories. In patients with pT1 cancer, neither intramural residual tumors nor intraoperative perforations were observed, and the LR rate was in the range of that associated with primary surgery. In patients with $\mathrm{pT} 2$ cancer, the risk of perforation increased, but the risk of residual tumor was low, and no LR was observed. Low rates of LR after completion surgery of pT1/2 tumors were also observed in most previous studies $[6,8,24,25,27-29]$. Only Gijn et al. [11] reported high LR rates in patients with pT2 cancer. In contrast, patients with pT3 cancer had the highest rates of residual tumors and perforations, which resulted in high LR rates. These rates exceeded the rates observed after primary surgery, even though the difference was not statistically significant. In these patients, endorectal sonography assessments had underestimated the stages. However, over time, as the quality of pretherapeutic imaging improved, the most advanced rectal cancers should be excluded from the local excision approach. It might also be important to consider the risk of intraoperative perforation for another group of patients: when a local excision is planned after CRT for advanced rectal cancers, patients with poor responses require special care to avoid intraoperative perforations.

Like LR, the rate of DM was associated with the pT and $\mathrm{pN}$ categories. Consequently, we found high CSS rates in patients with $\mathrm{pT} 1 / 2$ carcinomas, consistent with former studies [23]. In contrast, patients with pT3 cancers had the highest rates of involved lymph nodes and DM, which significantly lowered the CSS.

All the results of early radical surgery after a local excision were comparable to those of primary surgery, performed during the same time period. This result suggested that a delayed radical surgery after a local excision was not inferior to primary surgery, at least in pT1/2 cancers. Nevertheless, it should be emphasized that the pathological assessments revealed no residual tumors in most patients. To avoid a re-operation after a local excision, a meticulous local excision technique is essential to assure complete resection of all tumor tissues. Additionally, better pre-therapeutic diagnostic methods might clearly reveal the involvement of regional lymph nodes. This improvement is an important future aim that could increase the acceptance of local excision as a curative treatment for rectal cancer.

Our results could not answer the question of whether RT or CRT should be applied after a local excision and before radical surgery. In view of the $2.6 \%$ LR rate for all patients with $\mathrm{pT} 1 / 2$ tumors, the benefit of adjuvant therapy might be marginal. In patients with pT3 carcinomas, some results have indicated that a CRT before radical surgery could provide benefit [12], but in current practice, those patients are only seldom selected for local excision surgery.

This study had some limitations. First, it had a retrospective study design, and it was conducted in a single center with a long recruitment time. During that time, TEM was the preferred method of local excision, but TAMIS was introduced as an alternative method. Both methods appeared to provide comparable local excision quality [21]; consequently, the risks of complications after completion surgery that are due to the local excision technique should be comparable for both methods. Moreover, the quality of the TME resection was not determined at the beginning of the TME era. In most patients, no residual tumor was detected in resected specimens; thus, the actual impact of poor mesorectal quality after a completion TME remains unknown [9]. Prognostically, the more important risk factor was an intraoperative perforation in the rectal wall, which was always documented. All patients received open resections, but because laparoscopic and transanal methods (transanal total mesorectal excision) were introduced during the study period, the newer treatments might have conferred more advantages for patients treated later [30]. Finally, the numbers of patients included in the subgroups and the numbers of observed events were small, which limited the conclusions that could be drawn. On the other hand, all consecutive patients were included, and all patients were followed for a very long time period.

Our results suggested that performing completion surgery early after local excisions of pT1/2 rectal cancers with unfavorable tumor characteristics might not compromise the long-term oncological outcome. However, all efforts should be undertaken to avoid re-operations by applying 
thorough pre-therapeutic diagnostics and performing complete local excisions of early rectal cancers.

\section{Conclusions}

Rectal wall perforations at the local excision site and residual cancer were the main risks for poor oncological outcomes associated with completion surgery. Local excisions followed by early radical surgery did not appear to compromise outcomes compared to patients with primary surgery for $\mathrm{pT} 1 / 2$ rectal cancer. Improvements in clinical staging should allow more appropriate selection of patients that are eligible for a local excision of rectal cancer.

\section{Acknowledgements}

Not applicable

\section{Authors' contributions}

TJ contributed to the study concept and design, interpretation of data, and drafting of the article. UG contributed to the acquisition of data, interpretation of data, and revising of the article. MJ contributed to the acquisition of data and analyzing and revising of the article. $\Pi$ contributed to the acquisition of data, statistics, and interpretation of data. AH contributed to the study concept and design, interpretation of data, and revising of the article. DW contributed to the statistics and interpretation and revising of the article. All authors read and approved the final manuscript.

\section{Funding}

The authors confirm that they did not receive any grants, equipment, or support from any organizations

\section{Availability of data and materials}

The datasets used during the current study are available from the corresponding author on reasonable request

\section{Ethics approval and consent to participate}

All procedures performed in studies involving human participants were in accordance with the ethical standards of the institutional and/or national committee and with the 1964 Helsinki Declaration and its later amendments or comparable ethical standards. For this type of study, formal consent was not required.

Written informed consent was obtained from all patients after providing information about the study.

\section{Consent for publication}

Not applicable

\section{Competing interests}

The authors declare that they have no competing interests

\section{Author details}

${ }^{1}$ Department of General and Abdominal Surgery, University Medical Centre of the Johannes Gutenberg-University, Mainz, Germany. ${ }^{2}$ Department of General, Visceral and Vascular Surgery, Catholic Hospital, Mainz, Germany. ${ }^{3}$ Department of Heart, Chest and Vascular Surgery, University Medical Centre of the Johannes Gutenberg-University, Mainz, Germany. ${ }^{4}$ Institute of Medical Biostatistics, Epidemiology and Informatics, University Medical Centre of the Johannes Gutenberg-University, Langenbeckstr. 1, D 55131 Mainz, Germany.

Received: 20 May 2019 Accepted: 5 September 2019

Published online: 08 October 2019

\section{References}

1. Hermanek P, Gall FP. Early (microinvasive) colorectal carcinoma: pathology, diagnosis, surgical treatment. Int J Colorectal Dis. 1986;1:79-84.

2. Rullier E, Rouanet $P$, Tuech JJ, Valverde A, Lelong B, Rivoire M, et al. Organ preserveration for rectal cancer (GRECCAR 2): a prospective, randomized, open-label, multicenter phase 3 trial. Lancet. 2017;390:469-79.
3. Kumar AS, Coralic J, Kelleher DC, Sidani S, Kolli K, Smith LE. Complications of transanal endoscopic microsurgery are rare and minor: a single institution's analysis and comparison to existing data. Dis Colon Rectum. 2013;56:295-300.

4. Allaix ME, Rebecchi F, Giaccone C, Mistrangelo M, Morino M. Long-term functional results and quality of life after transanal endoscopic microsurgery. Br J Surg. 2011:98:1635-43.

5. Arezzo A, Passera R, Saito Y, Sakamoto T, Kobayashi N, Sakamoto N, et al. Systematic review and meta-analysis of endoscopic submucosal dissection versus transanal endoscopic microsurgery for large inivasive rectal lesions. Surg Endosc. 2014;28:427-38.

6. Yong Lee W, Lee WS, Yun SH, Shin SH, Chun HK. Decision for salvage treatment after trananal endoscopic microsurgery. Surg Endosc. 2007;21:975-9.

7. Baron PL, Enker WE, Zakowski MF, Urmacher C. Immediate vs. salvage resection after local treatment for early rectal cancer. Dis Colon Rectum. 1995:38:177-81.

8. Piessen G, Gabral C, Benovist S, Penna C, Nordlinger B. Previous transanal full-thickness excision increases the morbidity of radical resection for rectal cancer. Colorectal Disease. 2012;14:445-52.

9. Coton C, Lefevre JH, Debove C, Creavin B, Chafai N, Tiret E, et al. Does transanal local resection increase morbidity for subsequent total mesorectal excision for early rectal cancer? Colorectal Disease. 2019;21:15-22.

10. Morino M, Allaix ME, Arolfo S, Arezzo A. Previous transanal endoscopic microsurgery for rectal cancer represents a risk factor for an increased abdominperineal resection rate. Surg Endosc. 2013;27:3315-21.

11. Van Gijn W, Brehm V, de Graaf E, Neijenhuis PA, Stassen LP, Leijtens JW, et al. Unexpected rectal cancer after TEM: outcome of completion surgery compared with primary TME. EJSO. 2013;39:1225-9.

12. Gagliardi G, Newton TR, Bailey HR. Local excision of rectal cancer followed by radical surgery because of poor prognostic features does not compromise the long term oncological outcome. Colorectal Disease. 2013; 15:e659-e64.

13. Buess G, Mentges B, Manncke K, Starlinger M, Becker HD. Technique and results of transanal endoscopic microsurgery in early rectal cancer. Am J Surg. 1992;163:63-70.

14. Bouvet M, Milas M, Giacco GG, Cleary KR, Janjan NA, Skibber JM. Predictors of recurrence after local excision and postoperative chemoradiation therapy of adenocarcinoma of the rectum. Ann Surg Oncol. 1999;6:26-32.

15. Brodsky JT, Richard GK, Cohen AM, Minsky BD. Variables correlated with the risk of lymph node metastasis in early rectal cancer. Cancer. 1992; 69:322-6.

16. Morson BC, Bussey HJ, Samoorian S. Policy of local excision for early rectal cancer of the colorectum. Gut. 1977;18:1045-50.

17. UICC (Wittekind Ch., Greene FL., Henson DE., Hutter RVP., Sobin LH., eds.). TNM Supplement. A commentary on uniform use. 3rd ed. New York: John Wiley \& Sons; 2003

18. Borschitz T, Heintz A, Junginger T. The influence of histopathologic criteria on the long-term prognosis of locally excised pT1 rectal carcinomas: results of local excision (transanal endoscopic microsurgery) and immediate reoperation. Dis Colon Rectum. 2006:49:1492-506.

19. Team RC. R: a language and environment for statistical computing. $R$ Foundation for Statistical Computing. Vienna, Austria; 2014

20. Christoforidis D, Cho HM, Dixon MR, Mellgren AF, Madoff RD, Finne CO. Transanal endoscopic microsurgery versus conventional transanal excision for patients with early rectal cancer. Ann Surg. 2009;249:776-82.

21. Lee L, Edwards K, Hunter IA, Hartley JE, Atallah SB, Albert MR, et al. Quality of local excision for rectalneoplasms using transanal endoscopic microsurgery versus transanal minimally invasive surgery: a multiinstitutional matched analysis. Dis Colon Rectum. 2017;60:928-35.

22. Benson AB $3^{\text {rd }}$, Venook AP, Al-Hawary MM, Cederquist L, Chen YJ, Ciombor KK et al. (2018) Rectal cancer, version 2.2018, NCCN Clinical Practice Guidelines in Oncology. J Natl Compr Canc Netw 2018; 16: 874-901

23. Hompes R, McDonald R, Buskens C, Lindsey I, Armitage N, Hill J, et al. Completion surgery following transanal endoscopic microsurgery: assessment of quality and short- and long-term outcome. Colorectal Disease. 2013;15:e576-81.

24. Levic K, Bulut O. Hessefeldt, Bülow S. The outcome of rectal cancer after early salvage TME following TEM compared with primary TME: a casematched study. Techn Coloproctol. 2013;17:397-403.

25. Hahnloser D, Wolff BG, Larson DW, et al. Immediate radical resection after local excision of rectal cancer: an oncologic compromise? Dis Colon Rectum. 2005;48:429-37. 
26. Bülow S, Christensen U, Iversen LH, Harling H, Danish Colorectal Cancer Group. Intra-operative perforation is an important predictor of local recurrence and impaired survival after abdominoperineal resection for rectal cancer. Colorectal Dis. 2011;13:1256-64.

27. Peeters KC, Marijnen CA, Nagtegaal ID, Kranenbarg EK, Putter H, Wiggers T, et al. The TME Trial after a median follow-up of 6 years. Increased local control but no survival benefit in irradiated patients with resectable rectal carcinoma. Ann Surg. 2007;246:693-701.

28. Elmessiry MM, van Koughnett JAM, Maya A, DaSilva G, Wexner SD, Bejarano $P$, et al. Local excision of $\mathrm{T} 1$ and $\mathrm{T} 2$ rectal cancer: proceed with caution. Colorectal Disease. 2014;16:703-9.

29. Bach SP, Hill J, Monson JR, Simson JN, Lane L, Merrie A, et al. A predictive model for local recurrence after transanal endoscopic microsurgery for rectal cancer. Br J Surg. 2009;96:280-90.

30. Letarte F, Raval M, Karrimuddin A, Phang PT, Brown CJ. Salvage TME following TEM: a possible indication for TaTME. Tech Coloproctology. 2018; 22:355-61

\section{Publisher's Note}

Springer Nature remains neutral with regard to jurisdictional claims in published maps and institutional affiliations.

Ready to submit your research? Choose BMC and benefit from:

- fast, convenient online submission

- thorough peer review by experienced researchers in your field

- rapid publication on acceptance

- support for research data, including large and complex data types

- gold Open Access which fosters wider collaboration and increased citations

- maximum visibility for your research: over $100 \mathrm{M}$ website views per year

At $\mathrm{BMC}$, research is always in progress.

Learn more biomedcentral.com/submissions 